ORIGINAL ARTICLE

\title{
Urban youths' perspectives on violence and the necessity of fighting
}

\author{
S B Johnson, S Frattaroli, J L Wright, C B Pearson-Fields, T L Cheng
}

Injury Prevention 2004;10:287-291. doi: 10.1136/ip.2004.005793

See end of article for authors' affiliations

......................

Correspondence to:

Ms S B Johnson, $624 \mathrm{~N}$

Broadway, 5th Floor,

Baltimore, MD 21205 ,

USA; sjohnson@jhsph.edu
Objectives: To assess youth perceptions of the causes and consequences of violence generally, the causes and consequences of fighting specifically, and to determine how best to approach fighting in the context of violence prevention activities.

Methods: Thirteen structured focus group interviews with youths from three high violence urban settings: a large, urban high school, a training center for disadvantaged youths, and a school for adjudicated youths. Participants were 120 urban, predominately African-American youths and young adults ages 1422 years (mean: 17.2 years). Seven focus groups were conducted with females, and six with males.

Results: Adolescents identified the causes of violence on multiple levels including: individual, family, interpersonal, and community level factors. Most youths (89\%) had been in a physical fight. Participants felt that fighting was not "right", but identified situations in which it was necessary. Specifically, fighting was used as a problem solving tool, and could prevent escalation of violence. Youths felt that the adults in their lives, including physicians, were generally ill equipped to give advice about violence, as adults' experiences were so removed from their own. Participants looked to experienced role models to offer problem solving and harm reduction strategies. Youths were open to receiving anticipatory guidance about violence and fighting from primary care physicians they felt comfortable with, and who showed respect for their experiences.

Conclusions: Interventions that include blanket admonitions against fighting should be reassessed in light of youth perceptions that fighting plays a complex role in both inciting and preventing more serious violence. n 2001, 5385 youths aged 15-24 years died as a result of interpersonal violence in the United States. Homicide is the leading cause of death among African-Americans aged 1524 years, accounting for $40 \%$ of mortality. ${ }^{1}$ Further, in this age group, African-American youths are more than 6.5 times more likely to die from homicide than white youths. ${ }^{1}$ Despite substantial decreases in the national homicide rate in recent years, the toll of violence on urban youth remains enormous. ${ }^{2-6}$

Youths' experience with violence includes awareness of violent activity within their communities, and direct experience with interpersonal violence, particularly fighting. Nationally, $33 \%$ of high school students, including $43 \%$ of male and $24 \%$ of female students, report involvement in a physical fight in the preceding year. Intentional injury trends among urban adolescents suggest that although fighting has become less lethal, probably as a result of changing patterns of weapon use, the prevalence of fighting in high risk urban samples may be nearly double national estimates. ${ }^{78}$ The question emerges, therefore, how should fighting be addressed in prevention activities?

Despite its high prevalence, little is known about how youth understand fighting. Using focus groups, this study allowed us to examine violence and fighting from the perspective of urban youth. To design and evaluate effective violence prevention programs, practitioners and researchers must understand how youths view fighting and how best to approach fighting in the context of violence prevention activities. The purpose of this study was to understand and describe violence, fighting, and their prevention from the perspective of at-risk urban youth to help inform violence prevention theory and practice. This study was part of a larger program of emergency department surveillance and interviews designed to inform community based strategies for addressing adolescent assault injury.

\section{METHODS}

\section{Study design}

We conducted 13 focus group interviews ${ }^{10}$ with urban adolescents and young adults between 1996 and 1998 in Washington, DC. The first part of this study explored the youths' perceptions of the causes and consequences of violence in their lives and communities, and the strategies they used to stay safe. A second set of seven focus groups explored the most prevalent experience with interpersonal violence, fighting. In particular, this part of the study explored the causes and consequences of fighting, norms about fighting, and whom youth felt were credible sources of information about violence and fighting.

\section{Sites, recruitment, and participants}

We identified three study sites in Washington, DC that were likely to include urban youths who had witnessed or been involved with acts of violence. The sites included a large high school that had experienced several violent student deaths, a vocational training center for youths who had difficulty in traditional school settings for academic or behavioral reasons, and a school for adjudicated youths. We conducted six male focus groups, one at each of the sites, for both parts of the study. With female participants, we conduced three focus groups in part one (two at the high school and one at the training center) and four groups in part two (two at the high school and two at the training center).

At the two alternative schools, because of their small size, all students aged 14 years and older were invited to participate, and students who completed the consent process were included. At the high school, school administrators distributed consent forms to all students in several classes they selected; focus groups included all students aged 14 years and older who returned completed consent forms. We obtained parental consent for minor students, and all students completed an 
assent process. Each participant received \$20 for participating. The Children's National Medical Center institutional review board approved the study protocol.

\section{Procedures}

We created two semistructured focus group interview guides, one for each part of the study. The first guide explored definitions, types, and causes of violence, strategies for staying safe, and suggestions for violence prevention interventions. The second guide probed social norms about fighting, consequences of fighting, instances where fighting was necessary, and whose opinion mattered about fighting, with a particular focus on the role of physicians. Focus group moderators and observers, who were members of the project staff, received six hours of training on the use of the guides by a qualitative methodologist. The guides were pilot tested with a group of urban peer health educators.

Groups ranged in size from 6-10 participants and lasted 1.5-2 hours. Each group was sex segregated, and facilitated by a same sex moderator and an observer. Moderators used the protocols to ask open ended questions, and to probe responses. All focus groups were audiotaped and transcribed. Observers took notes and documented non-verbal aspects of the discussions. Before each group, participants completed a brief survey that included demographic information, and whether they had ever carried a gun or other weapon, been in a physical fight, or had a fight related injury.

\section{Analysis}

We used $\chi^{2}$ difference of proportion tests to identify differences in focus group composition that might influence our analysis. Next, we used a three step process to analyze the focus group data. Firstly, an experienced primary coder (SJ) open coded data and consulted with a secondary coder (SF), an expert in qualitative methodology. Next, we conducted detailed coding and developed a coding manual. Finally, we conducted thematic analysis of the coded data to identify patterns and themes. We reconciled differences in thematic organization through discussion among authors (SJ, SF, TC). We examined data from parts one and two separately, and reported the findings for each. We then combined the data from the two parts for the discussion, as many of the emerging themes were related and complementary. Preliminary analyses of the data by subgroup (sex, site) revealed remarkable consistency across subgroups, making stratified analyses uninformative. This analysis is based on survey data, focus group transcripts, and observers' notes, which provided situational and contextual detail.

\section{RESULTS}

A total of 120 youths aged 14-22 years (mean 17.2 years) participated in the 13 focus groups. Ninety seven percent of participants were African-American, and 44\% were male. Participants in part one were somewhat younger (mean 16.8 $v 17.5$ years; $\mathrm{p}=0.03$ ) and more likely to have carried a weapon $(68.4 \% \vee 45.9 \% ; \mathrm{p}=0.01)$ than those in part two. Participant sociodemographic characteristics are summarized in table 1. A majority of participants had carried a weapon (57\%), 29\% had carried a gun, and $89 \%$ had been in a physical fight.

\section{RESULTS FROM PART 1 \\ Causes of violence}

The youths talked about violence in broad terms; no predominant cause of violence emerged from the discussion. They identified individual level determinants of violence such as stress and substance abuse, and family factors such as witnessing violence, lack of "home training", and family norms supportive of violence. Interpersonal causes of violence

\begin{tabular}{|c|c|c|}
\hline Characteristic & No & $\%^{*}$ \\
\hline \multicolumn{3}{|l|}{ Age (years) } \\
\hline $14-16$ & 46 & 39 \\
\hline $17-19$ & 62 & 53 \\
\hline $20-22$ & 10 & 9 \\
\hline \multicolumn{3}{|l|}{ Sex } \\
\hline Male & 53 & 44 \\
\hline Female & 67 & 56 \\
\hline \multicolumn{3}{|l|}{ Grade in school } \\
\hline 8th & 1 & 1 \\
\hline 9 th & 5 & 4 \\
\hline 10th & 32 & 27 \\
\hline 11 th & 33 & 28 \\
\hline 12 th & 11 & 9 \\
\hline Othert & 35 & 30 \\
\hline \multicolumn{3}{|l|}{ Race/ethnicity } \\
\hline Black/African-American & 116 & 97 \\
\hline Hispanic & 2 & 2 \\
\hline White & 1 & 1 \\
\hline Asian & 1 & 1 \\
\hline \multicolumn{3}{|l|}{ Ever carried a gun } \\
\hline Yes & 35 & 29 \\
\hline No & 84 & 71 \\
\hline \multicolumn{3}{|l|}{ Ever carried a weapon } \\
\hline Yes & 67 & 57 \\
\hline No & 51 & 43 \\
\hline \multicolumn{3}{|l|}{ Ever been in a physical fight } \\
\hline Yes & 105 & 89 \\
\hline No & 13 & 11 \\
\hline \multicolumn{3}{|c|}{ Ever received medical treatment for fight injury } \\
\hline Yes & 22 & 19 \\
\hline No & 95 & 81 \\
\hline \multicolumn{3}{|c|}{ Mother's highest level of education } \\
\hline Did not finish high school & 21 & 18 \\
\hline High school diploma/GED & 46 & 39 \\
\hline Some college or more & 35 & 30 \\
\hline Unknown/other & 16 & 14 \\
\hline
\end{tabular}

included standing up for friends, jealousy, misunderstandings, disrespect, and gossip. Participants emphasized that the desire for retaliation fuelled continued violence. In addition to interpersonal factors, participants identified community level factors including drug selling and neighborhood "beefs" (disagreement) over territory. Participants also cited boredom and lack of opportunity in their neighborhoods as a cause of violence, as exemplified in a comment by one youth: "when me and my boys are chillin', we don't really have a planned violence, we, like, get bored to do something".

\section{Staying safe}

Participants described a variety of strategies for avoiding violence. They identified individual strategies (such as minding your own business and carrying a weapon), interpersonal strategies (such as cultivating a tough image and hanging around in groups), and community strategies (such as building relationships with others in your neighborhood). One participant commented that feeling isolated from her neighborhood made her feel vulnerable to violence: "I don't go outside that much and the people around my neighborhood, most of them did not meet me until this year because I will not go outside and talk to them. It's like, I'm not anybody they know, they not gonna protect me".

\section{Barriers to violence prevention: lack of knowledgeable role models}

Youth reported that adult role models were generally available, appreciated, and respected; however, they often 
saw adults' violence related advice and guidance as inappropriate and based on outdated, incomplete understandings. Adults "need to know that a whole lot of things have changed from the days [when] they were in school ... I listen to some of the stuff my grandmother used to say to me, and I be like, 'well it don't work like that no more"'. Participants also expressed frustration that their expertise and contributions were not acknowledged by adults, "they figure "you young and you don't know what's going on', but we see more than what they think we see. We know more than what they think we know. We're out doing positive things ...". With the idea that youths' expertise could be used to understand and address fighting, the second part of the study addressed the type of violence they reported direct involvement with.

\section{RESULTS FROM PART 2}

\section{Shared social norms about fighting}

Participants identified situations in which fighting ("wrecking") was necessary. Most prominently, youth felt they had to fight when they, or their friends, were physically attacked. Participants' attitudes about fighting often explicitly reflected their parents' teaching. "Like your mother used to say, somebody hit you, you better hit "em back".

Youths differentiated between situations that legitimately called for violence, such as standing up for a friend, and "petty" reasons for fights: "if somebody come up to you like'I heard he was talking about your mother', or 'he was talking about your girl', man, that's petty stuff. You don't have to go on no rampage off of something that you heard". Participants acknowledged that fighting was never "right", and "everybody knows" that you should walk away; nonetheless, they asserted fighting was sometimes necessary. Participants recognized that mainstream cultural values against fighting did not always apply to them, given the violent realities of their lives. A participant observed that those who hadn't "been raised around all that violence" were the only youths who could realistically walk away from a fight.

\section{Potential benefits of fighting}

Participants described some physical and social risks to fighting (that is, that fighting could make you look like a troublemaker), but they also identified significant benefits including revenge, respect, popularity, and social power. "If you fight somebody and beat them up, it's like everybody wants to be your friend, or everybody knows your name that didn't speak to you before-they speak to you now". Fighting could also be cathartic, a way of letting go of stress and coping with anxiety: "when you fight you got, like, so much stress inside of you that you just have to get rid of it, and once you get rid of it on that person that you angry at, it's gone and you be relaxed and you can chill".

One of the most consistent focus group findings was that fighting could prevent further, potentially more serious violence, and could be used as a problem solving tool. Youths felt they could only avoid fights until they were forced to defend themselves. If they chose not to fight, aggressors would continue to bully them until they stood up for themselves. Further, participants argued if they chose to avoid a fight, they risked having the conflict escalate over time: "you wanna fight me, I'm gonna get it over with, cause they gonna keep on goin' and goin'... cause if you don't settle that ... it's gonna keep on escalatin". Youths explained that walking away from a fight could be counterproductive or even dangerous, if, as some participants warned, their aggressors returned with weapons.

\section{Violence and fighting: whose opinion matters?}

Participants suggested they would listen to family members, close friends, or a sympathetic teacher or counsellor's advice about violence out of respect. Most acknowledged, however, that if they were advised not to fight, it would not necessarily deter them. Participants felt those most qualified to counsel them about violence prevention were individuals with similar life experiences, although these were rarely the people who offered them advice.

Most participants had never talked to a doctor about violence, and were divided about whether or not a doctor's opinion mattered. Youths felt the physician's role in violence prevention counselling was to link violence to its direct health effects, or to highlight the potential for violent behavior to exacerbate existing health problems. In general, doctors were not credible sources of information on violence. They perceived clinicians as rushed and difficult to talk to, often using big words. Emergency physicians were seen as particularly unsuited to anticipatory guidance about violence and fighting; however, participants cited their primary care physicians, especially those with whom they had good relationships, as possible sources of support. Participants suggested that physicians could improve their credibility with teenagers by showing respect for their life experiences and listening to them during medical visits.

\section{DISCUSSION}

Participants often referred to lethal and serious incidents when they discussed violence, although few adolescents had been involved in such events personally. In contrast, the majority of participants reported having been in a physical fight. Approximately one in five had ever sought medical attention for a fight related injury. Because avoiding a fight could lead to escalation of violence or weapon involvement, participants saw fighting, in some circumstances, as a harm reduction strategy. Further, they acknowledged the dissonance they felt between mainstream cultural norms shunning fighting, and the normative and possibly even protective nature of fighting in their own experience. This conflict between personal experience and advice led youths to question the credibility of many adults on violence related topics. They sought "experienced" role models with first hand knowledge of their problems, who could offer solutions relevant to their lives.

The literature on youth violence focuses on lethal and serious incidents, ${ }^{11}$ although relatively few adolescents are personally involved in such violence. As most fights do not result in medical treatment, they risk going unnoticed by medical and public health professionals. These results help to identify cases in which fights might escalate dangerously, such as when fights are initially avoided, fuelling a persistent instigator's anger and resolve. Further, assault injury is a risk factor for repeat injury. ${ }^{12}{ }^{13}$ Fighting provides an opportunity to use teenagers' experiences to understand and address an important subset of interpersonal violence. Scant literature has taken into account the youth perspective on violence. ${ }^{14}{ }^{15}$ However, such research provides important social context for prevention activities. In this study, participants' sophisticated understanding of the complex causes of violence at the individual, interpersonal, family, and community levels, lends credibility to their advice about how to address the violence in their lives.

The prevalence of fighting among participants supports the contention that fighting may be normative in this population. Similarly, our results cast doubt on the utility of prevention programs that rely on blanket prohibitions against fighting. Programs that emphasize "turning the other cheek" in the face of a fight, or focus on building conflict resolution skills without acknowledging the benefits youth perceive from fighting, are unlikely to be effective in isolation. In violent urban settings, such admonitions 
may be in direct conflict with shared norms about fighting Participants' insights reveal the complexities involved in fighting in that it may lead to injuries, but also, in some cases, may be protective against further, more serious, forms of violence. At a minimum, violence prevention programs should acknowledge and address the social and physical benefits of engaging in violent behavior that may accrue to youths.

Participants in this study defied the stereotype that urban youth lack concerned adults who are looking out for them. Also, by requesting role models with similar experiences to step forward with advice and guidance about violence, they were indirectly acknowledging the importance of their own lived experience. A recent study by Sheehan and colleagues suggests that preadolescent urban youth look to trusted adults to help keep them safe from violence. ${ }^{15}$ Our findings indicate that, with age, youths may come to trust the adults in their lives less, and develop their own strategies for staying safe. When available, peers with similar life experiences may inform those strategies.

This study helps to clarify youths' perceptions of the physician's role in violence prevention anticipatory guidance and builds on previous work in this area. In particular, these findings support research by Dowd and colleagues that suggests that youths do not see the emergency department and emergency physicians as well suited to violence prevention counseling. ${ }^{16}$ However, the current study identifies some settings where youths might be more receptive to anticipatory guidance about violence. Specifically, youths are interested in talking about violence with a primary care physician who knows them, shows respect for their experiences, and acknowledges that cultural norms about fighting may be different in their communities. These findings suggest that physicians, recognizing the contextual realities of urban youths' lives, may need to counsel them about harm reduction strategies associated with fighting, such as discouraging weapon use. In addition, clinicians, public health professionals, and other violence prevention advocates should look for opportunities to link youths with experienced role models whose advice is most likely to be heeded.

As a qualitative, theory building study, this design did not produce findings that are generalizable to the population level. However, this approach allowed us to reveal and explore complexities of the topic that might have been overlooked using traditional quantitative survey methodology. Further, the consistency of findings across sites and sexes suggests these results accurately represent the experiences and insight of the participants.

\section{Key points}

- Urban youths have a sophisticated understanding of the multilevel causes of violence including individual, family, and community factors.

- Fighting may be used as a problems solving tool and may help youths avoid more serious incidents of violence.

- Urban youths are most likely to listen to advice about violence and fighting if it comes from individuals with life experiences similar to theirs.

- Prevention programs that discourage fighting should be aware of the complex role fighting plays in the lives of these youths.

\section{CONCLUSION}

This study serves as a first, exploratory step in the research process. Further studies could elucidate how violence and fighting are perceived and experienced across different subpopulations of youth. Strategies youths identified for addressing violence and fighting, including using experienced role models and primary care physicians to deliver violence prevention messages, could be tested empirically. Further research could also help identify other circumstances under which unarmed fighting escalates to more serious incidents of violence. Finally, our findings suggest opportunities for primary prevention, particularly by addressing structural factors that the youths identified, such as community cohesion. This study shows that adolescents' sophisticated understanding of the realities of violence in their lives can help to inform violence prevention programs that are most likely to resonate with this group.

\section{ACKNOWLEDGEMENTS}

This project was supported by the Centers for Disease Control and Prevention R49/CCR331657 and the Robert Wood Johnson Foundation Generalist Faculty Scholar Program (TLC).

The authors wish to thank Bill McKinney for his assistance with the focus groups, as well as the youths who participated in the groups.

\section{Authors' affiliations}

S B Johnson, S Frattaroli, The Johns Hopkins Bloomberg School of Public Health, Baltimore, Maryland, USA

J L Wright, Division of Emergency Medicine and Trauma Services, Children's National Medical Center, and George Washington University Schools of Medicine and Public Health, Washington, DC, USA

C B Pearson-Fields, Mautner Project, Washington, DC, USA

T L Cheng, Division of General Pediatrics and Adolescent Medicine, Johns Hopkins University School of Medicine, Baltimore, Maryland, USA

\section{REFERENCES}

1 Centers for Disease Control and Prevention. Web-Based Injury Statistics Query and Reporting System (WISQARS). Available at: http://www.cdc.gov/ ncipc/osp/data.htm (accessed 2 March 2004)

2 Mazza JJ, Reynolds WM. Exposure to violence in young inner-city adolescents: relationships with suicidal ideation, depression, and PTSD symptomatology. J Abnorm Child Psychol 1999:27:203-13.

3 US Department of Health and Human Services. Youth violence: a report of the Surgeon General. Rockville, MD: US Department of Health and Human Services, Centers for Disease Control and Prevention, National Center for Injury Prevention and Control, Substance Abuse and Mental Health Services Administration, Center for Mental Health Service, and National Institutes of Health, National Institute of Mental Health, 2001.

4 McGee ZT, Davis BL, Brisbane T, et al. Urban stress and mental health among African-American youth: assessing the link between exposure to violence, problem behavior, and coping strategies. J Cult Divers 2001;8:94-104.

5 O'Keefe M. Adolescents' exposure to community and school violence: prevalence and behavioral correlates. J Adolesc Health 1997; 20:368-76.

6 Schwab-Stone M, Chen C, Greenberger E, et al. No safe haven II: the effects of violence exposure on urban youth. J Am Acad Child Adolesc Psychiatry 1999;38:359-67

7 Cheng TL, Wright JL, Fields CB, et al. Violent injuries among adolescents: declining morbidity and mortality in an urban population. Ann Emerg Med 2001;37:292-300

8 Grunbaum JA, Kann L, Kinchen SA, et al. Youth risk behavior surveillanceUnited States, 2001. MMWR Surveill Summ 2002;51:1-62.

9 Morgan D. Focus groups as qualitative research. 2nd Ed. Thousand Oaks, CA: Sage Publications, 1997.

10 Stewart D, Shamdasani P. Focus groups: theory and practice. Newbury Park, CA: Sage Publications, 1990.

11 Prothrow-Stith D. Murder is no accident: understanding and preventing youth violence in America. San Francisco: Jossey-Bass, 2004.

12 Cheng TL, Schwarz D, Brenner RA, et al. Adolescent assault injury: risk and protective factors and locations of contact for intervention. Pediatrics 2003; 112:931-8 
13 Redeker N, Smeltzer S, Kirkpatrick J, et al. Risk factors of adolescent and young adult trauma victims. Am J Crit Care 1995;4:370-8.

14 Rich J, Stone D. The experience of violent injury for young AfricanAmerican men: the meaning of being a "sucker". J Gen Intern Med $1996 ; 11: 77-82$.
15 Sheehan K, Kim LE, Galvin JP Jr. Urban children's perceptions of violence. Arch Pediatr Adolesc Med 2004;158:74-7.

16 Dowd MD, Seidel JS, Sheehan K, et al. Teenagers' perceptions of personal safety and the role of the emergency health care provider. Ann Emerg Med 2000;36:346-50.

\section{Cochrane Injuries Group}

he latest systematic reviews produced by reviewers working with the Cochrane Injuries Group (CIG), and published on the Cochrane Library, include one on an important prevention topic - the prevention of burns and scalds in children. Such injuries are of course a significant cause of morbidity and mortality. ${ }^{1}$ The review also addresses the need to determine whether injury countermeasures that are accepted as being effective can be successfully rolled out in the wider community.

The reviewers who took on this task are based at Injury Prevention and Control Australia and the School of Population Health, University of Queensland, Australia. After a comprehensive search, they only found three studies that met their criteria for inclusion. Only one of these showed a significant decrease in paediatric burn and scald injury in the intervention community compared with the control community. The reviewers have concluded: "There are a very limited number of research studies allowing conclusions to be drawn about the effectiveness of community-based injury prevention programmes to prevent burns and scalds in children ... It is important that a framework for considering the problem of burns/scalds in children from a prevention perspective be articulated, and that an evidence-based suite of interventions be combined to create programme guidelines suitable for implementation in communities throughout the world".

As is the case with most Cochrane reviews, the results highlight the need for further research and it is to be hoped that they will influence the direction that such research will take. It is also worth noting that the three included studies in the review were all conducted in developed countries (the USA or Norway). Most of the global fire related burn injury burden, nevertheless, falls on developing countries. ${ }^{2}$ Financial and other constraints may make it impossible to implement in these countries interventions that look promising in high income nations. It is essential to determine what interventions are effective in cutting injury rates in "the majority world".

More CIG reviews are on the way-some on the treatment of injury and some on prevention. One of our latest review protocols is on a prevention topic-motorcycle helmet legislation. ${ }^{3}$ The authors are again based in Australia, a country where injury prevention research and Cochrane Collaboration activities are both at encouragingly high levels. The reviewers set out on their project at a time when many countries around the world still lack legislation requiring motorcyclists to wear helmets. Indeed, many states in the USA have repealed or weakened their helmet legislation. The review will examine the effect of motorcycle helmet legislation on death and injury rates in motorcyclists, and on helmet wearing rates.

Among recently registered CIG titles, for which protocols have yet to be published are: "Home safety education for injury prevention", "Interventions for reducing the use of babywalkers", "Community-based interventions for preventing alcohol-related injuries", and "Interventions for drowning prevention".

We invite you to comment on our systematic reviews and to suggest topics that we should address in the future. New reviewers are also welcome; you don't have to be Australian!

P I Chinnock

Cochrane Injuries Group; paul.chinnock@|shtm.ac.uk (www.cochrane-injuries.Ishtm.ac.uk) Competing interests: Paul Chinnock is employed by the London School of Hygiene, on a grant from the UK NHS, to be the Managing Editor of the Cochrane Injuries Group.

\section{REFERENCES}

1 Turner C, Spinks A, McClure R, et al. Community-based interventions for the prevention of burns and scalds in children (Cochrane review). In: The Cochrane Library, ed. Issue 3. Chichester, UK: John Wiley, 2004. An abstract of this review may seen (free access) on www.update-soltware.com/abstracts/ab004335.htm.

2 World Health Organisation. The injury chartbook: a graphical overview of the global burden of injuries. Geneva: WHO, 2002.

3 Ivers RQ, Blows S, Liu BC, et al. Motorcycle helmet legislation for preventing injuries in motorcyclists (protocol for a Cochrane review). In: The Cochrane Library, ed. Issue 3. Chichester, UK: John Wiley, 2004. 